\title{
Probe Design to Robustly Locate Anatomical Features
}

\author{
Kevin B. Inkpen, Richard J. Emrich, Antony J. Hodgson \\ Department of Mechanical Engineering \\ University of British Columbia, Vancouver, BC, Canada
}

\begin{abstract}
Computer-assisted surgical techniques which seek to avoid relying on CT or MRI scans often require intraoperative location of anatomical features. The conventional optoelectronic probe measures a cloud of points on the feature surface, but the resulting location estimate is subject to bias and variation due both to local deformations in the surface and to measurement noise. We compare three probe designs - the conventional point probe, a flat probe and a V-probe - and show that all exhibit strong directional variability when estimating the centre of a quarter arc. We also show that the $\mathrm{V}$-probe design is superior in tests on a $2 \mathrm{D}$ image, reducing the variability in localizing a femoral condyle by $50 \%$.
\end{abstract}

\section{Introduction}

We are currently developing a technique for computer-assisted total knee replacement (TKR) surgery which does not require preoperative CT scans (similar to [1]). Certain variations of our approach require approximating centres of the posterior portions of the femoral condyles, which have been shown to closely fit spherical surfaces [2]. We propose to pass an optoelectronic digitizing probe over the condylar surfaces and fit a sphere to them, taking the centre of the fitted sphere as the condylar centre. This paper concentrates on the reliability of this latter process. In particular, we wish to characterize the repeatability with which we can define these centres.

Commercially available probes usually have a point or small (about $2 \mathrm{~mm}$ diameter) spherical end that touches the subject (see Figure 1). Such probes are versatile in that they can be used define points on surfaces with detailed concave and convex features. In our application, however, the subject surface is generally convex with local flaws that do not represent the ideal sliding surface that we are trying to locate. We suggest alternative probe designs that may be less sensitive to local deformations in the articular surface and may lead to more reliable estimates of the condylar centres. For general registration applications as discussed in [3], the effect of probe design should be considered when the goal is to quickly gather data that accurately constrain a convex feature.

In this paper we investigate using a probe with a flat contact surface to provide data as a series of tangent lines (rather than points) as the probe is swept along a 
surface. We also investigate the use of a V shaped probe that returns a series of local curvature bisector lines from a similar scan. A best fit 'centre' point of the contour can be found by minimizing an appropriate cost function. At this stage, we are interested in the repeatability of an estimated centre point location found from a single scan of a surface.

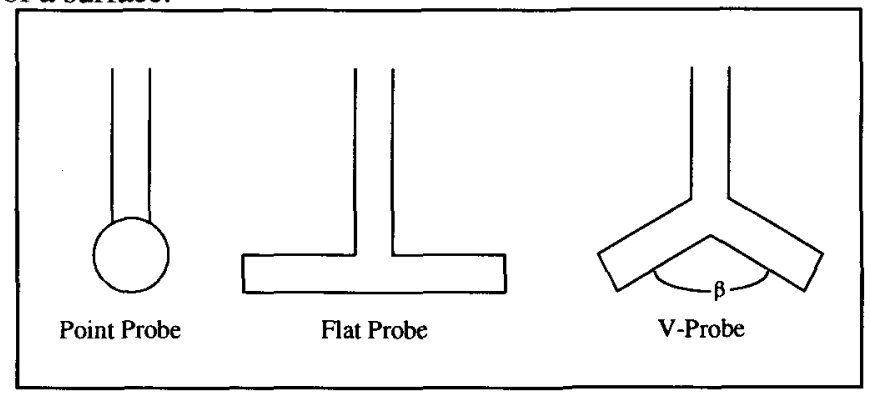

Fig. 1. Different Probe Designs

We simulate 1000 scans each over two different $2 \mathrm{D}$ test curves using each probe design and compare the standard deviations from the mean of the resulting estimated centres. We show that the V-probe exhibits significantly less standard deviation, thus better repeatability, than the other two designs.

\section{Methodology}

We simulated tests of a point probe, a flat probe, and $V$-probes with a variety of $\beta$ angles $\left(10^{\circ}\right.$ increments from $120^{\circ}$ to $\left.170^{\circ}\right)$ on two reference curves:

(1) the circumference of a true quarter arc with a $13 \mathrm{~mm}$ radius.

(2) the posterior-distal quadrant of a 2D contour of the lateral condyle of a human femur ( $13 \mathrm{~mm}$ radius) obtained from a sagittal MRI of a 31 year old female with no significant knee pathology.

For both curves, we simulated sweeping the probes across a $90^{\circ}$ range, added measurement noise, and found the best-fit centre point by minimizing an appropriate cost function (see Sections 2.1 - 2.3) using MATLAB's Nelder-Mead simplex method.

For each probe type and each reference curve, we ran 1000 simulated scans which resulted in 1000 estimated centres. We then computed the standard deviation of centre point location as a measure of the repeatability of the probe type.

We simulated the intraoperative process of acquiring points along the curve as a rotation of the probe about the origin (roughly the centre of the curve) with the radius being determined by the requirement that the probe maintain contact with the curve. In practice the surgeon would tend to start with the probe at one end of the curve with zero velocity, sweep through approximately $90^{\circ}$, and come to rest at the end of the curve. We therefore calculated the sampling position vector $\theta$ as: 


$$
\theta=\theta_{s}+\left(\theta_{f}-\theta_{s}\right)\left(1+\sin \left(\pi\left(\left(t / t_{s}\right)-0.5\right)\right)\right) / 2
$$

where $t$ is a vector of time steps from $t=0$ to total scanning time $\left(t_{s}\right)$ with a step size of (sampling frequency) ${ }^{-1} . \theta_{s}$ and $\theta_{f}$ are the start and finish angles of the scanned range. In this study $t_{s}=1 \mathrm{~s}$, sampling freq. $=60 \mathrm{~Hz}, \theta_{s}=90^{\circ}$, and $\theta_{\mathrm{f}}=180^{\circ}$ for all simulations and all angles are measured positively $\mathrm{CCW}$ from the $\mathrm{x}$ axis. For the $\mathrm{V}$ probes, the range of $\theta$ was reduced to $\left[\theta_{\mathrm{f}}-(\pi-\beta) / 2\right]-\left[\theta_{\mathrm{s}}+(\pi-\beta) / 2\right]$ to ensure that there was no contact outside of the sampled range used for the other probes.

During each simulation, we added white noise with $\sigma=0.02$ radians to each sampling position $\theta$ to ensure that we were not always sampling at the same points. We also added white noise with $\sigma=0.2 \mathrm{~mm}$ to all $(\mathrm{x}, \mathrm{y})$ coordinate data to simulate the measurement errors of a typical optoelectronic localizer. Assuming a distance of $120 \mathrm{~mm}$ from the probe surface to the probe markers, a corresponding white noise with $\sigma=0.20 / 120=0.0017$ radians was added to all angular data.

\subsection{Point Probe}

To estimate the centre of the best-fit circle, we computed the contact point of the probe to the surface at each sampling position $\theta$ and added noise as described above, producing a set of noisy data points $\left(x_{p}, y_{p}\right)$. With candidate circles defined by their centre coordinates and radius $\left(x_{c}, y_{c}, r_{c}\right)$, the point probe cost function 'PPCF' is the sum of squared normal distances from the points to the candidate circle:

$$
P P C F=\Sigma\left(\mathrm{r}_{\mathrm{c}}-\left(\left(\mathrm{x}_{\mathrm{p}}-\mathrm{x}_{\mathrm{c}}\right)^{2}+\left(\mathrm{y}_{\mathrm{p}}-\mathrm{y}_{\mathrm{c}}\right)^{2}\right)^{1 / 2}\right)^{2}
$$

\subsection{Flat Probe}

For the flat probe, we calculated the contact point between the probe and the surface contour, $\left(x_{f}, y_{f}\right)$, and the angle of the probe face, $(\alpha)$, at each sampling position $\theta$ and added noise. The flat probe cost function 'FPCF' is the sum of squared distances along the lines coperpendicular to both the candidate circle and the flat probe:

$$
F P C F=\Sigma\left(\left(\mathrm{x}_{\mathrm{f}}-\mathrm{x}_{\mathrm{f}}\right) \sin \alpha+\left(\mathrm{y}_{\mathrm{f}}-\mathrm{y}_{\mathrm{l}}\right) \cos \alpha\right)^{2}
$$

where $\left(x_{t}, y_{t}\right)$ are the co-ordinates of the point on the candidate circle whose tangent is parallel to the probe face.

\subsection{V-Probe}

At each sampling position $\theta$, the $\mathrm{V}$-shaped probe contacts the surface contour in two places and the centre of any 'local' best-fit circle at this sampling position must lie somewhere along the bisector of the V. As the probe is swept along the surface 
contour over the scanned range, these bisectors form a set of lines intersecting near the centre of an overall best-fit circle. The angle of each bisector, $\gamma$, and a point on the bisector at the apex of the $\mathrm{V},\left(\mathrm{x}_{v}, \mathrm{y}_{v}\right)$, form the data set and have noise applied. The cost function 'VPCF' expresses the sum of the squared normal distances between the candidate centre point, $\left(\mathrm{x}_{\mathrm{c}}, \mathrm{y}_{\mathrm{c}}\right)$, and the bisectors:

$$
V P C F=\Sigma\left(\left(\mathrm{x}_{\mathrm{v}}-\mathrm{x}_{\mathrm{c}}\right) \sin \gamma-\left(\mathrm{y}_{\mathrm{v}}-\mathrm{y}_{\mathrm{c}}\right) \cos \gamma\right)^{2}
$$

Note that in this case, we cannot explicitly estimate the radius of the arc.

\section{Results}

\subsection{True Arc}

Figures 2 and 3 show the 1000 estimated arc centres for the point probe and $140^{\circ} \mathrm{V}$ probes respectively. The results for the flat probe are comparable to the point probe and so are not shown here. Note the extended distribution of these estimates along the axis of symmetry of the arc.

To evaluate the repeatability of each probe design, standard deviations in centre point location were found for two different measures: 'Sym. Axis' is the standard deviation measured parallel to the bisector of the scanned range. 'Perp. Axis' is the standard deviation measured perpendicular to the bisector of the range.

Table 1. Standard deviations of centre point location from true arc

\begin{tabular}{|c|c|c|}
\hline & Sym. Axis (mm) & Perp. Axis (mm) \\
\hline Point & 0.134 & 0.043 \\
\hline Flat & 0.116 & 0.041 \\
\hline V120 & 0.137 & 0.025 \\
\hline V130 & 0.112 & 0.027 \\
\hline V140 & 0.080 & 0.027 \\
\hline V150 & 0.070 & 0.028 \\
\hline V160 & 0.062 & 0.030 \\
\hline V170 & 0.056 & 0.028 \\
\hline
\end{tabular}

\subsection{Posterior-Distal Condyle Image}

As in the first test, 1000 scans were simulated with each probe design on the same set of points representing the contour of a sagittal section through the distal femur. All three probe designs proposed a different mean centre point location (See Fig. 4). 


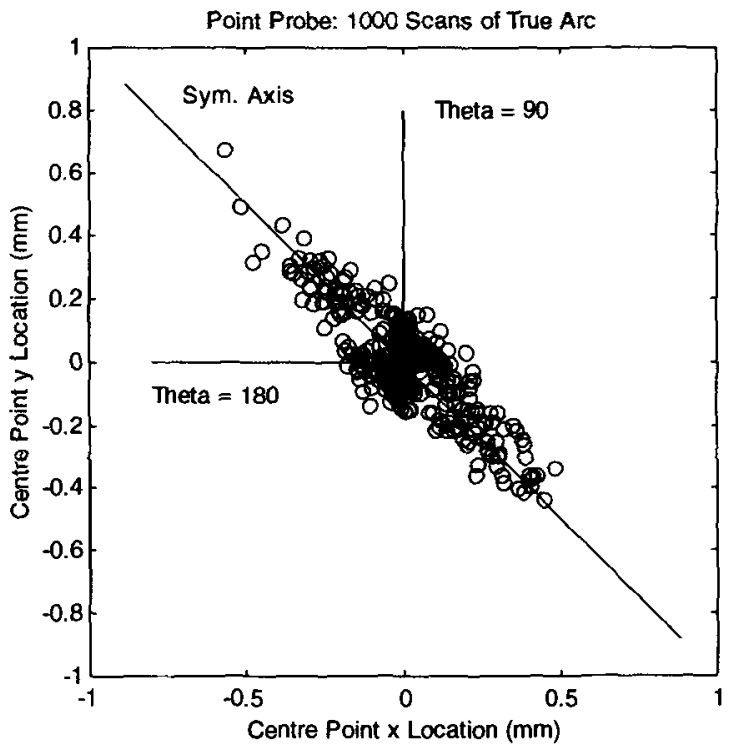

Fig. 2. Point probe: Estimated centre point locations for true arc.

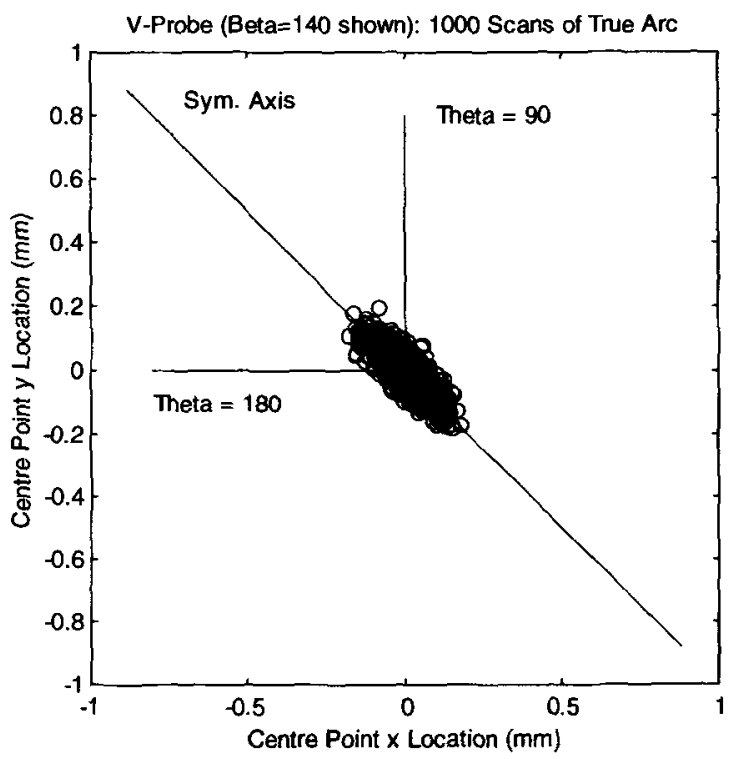

Fig. 3. V140 Probe $\left(\beta=140^{\circ}\right)$ : Estimated centre point locations for true arc. 


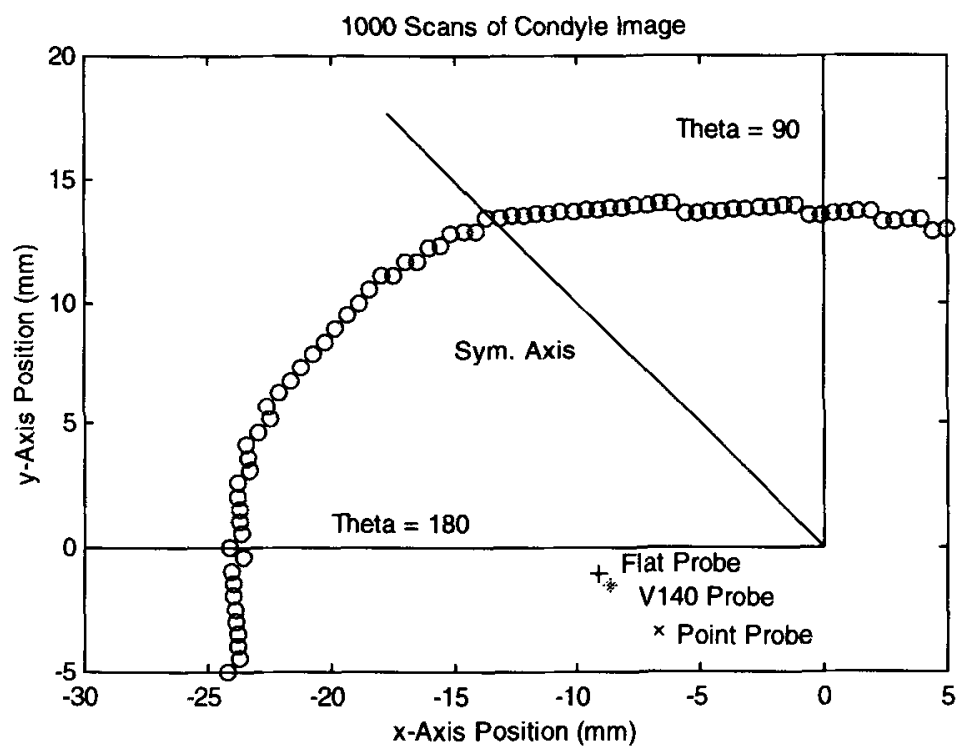

Fig. 4. Average centre point estimation for each probe design on condyle image.

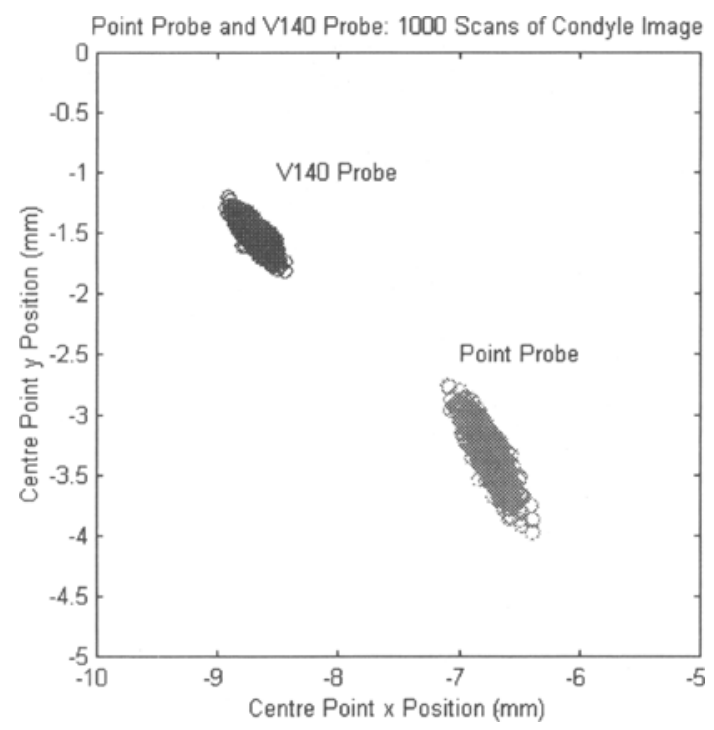

Fig. 5. Distribution of centre point estimates for point and V140 probes on condyle image.

Repeatability of each probe design was calculated again and is shown in Fig. 6. 


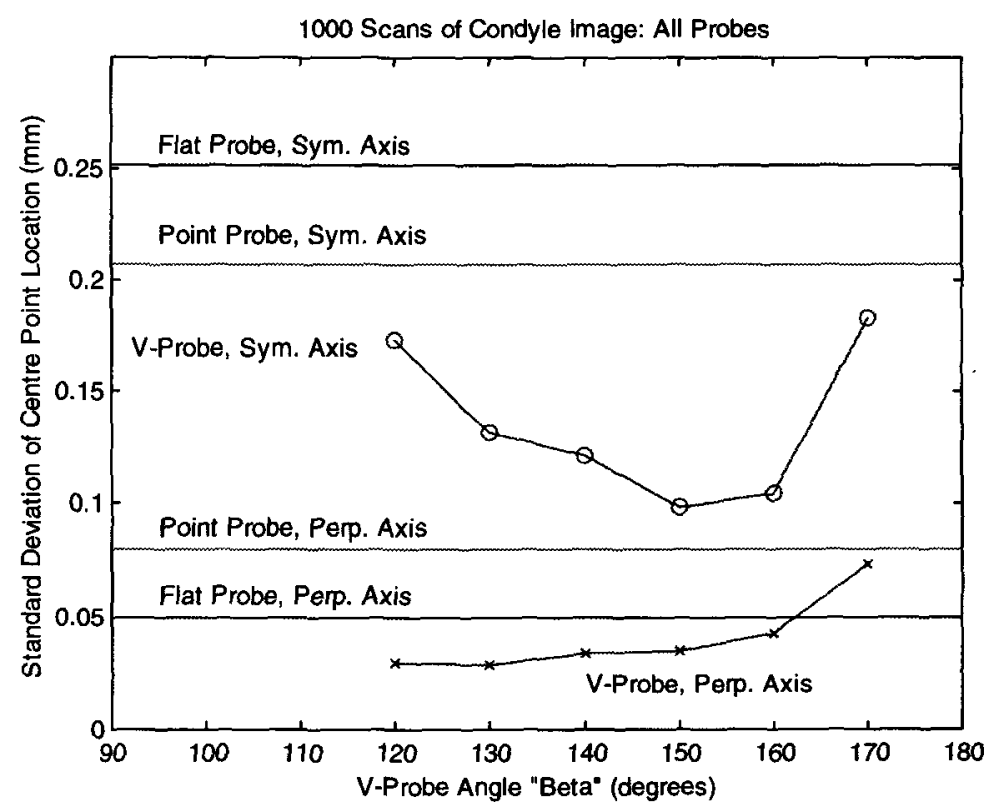

Fig. 6. Standard deviation of centre point location on condyle image, all probe designs.

\subsection{Discussion}

The extent of the cloud of centre point estimates derived from the true arc indicates sensitivity to measurement noise. All three probes exhibited 2-5X more deviation along the arc's axis of symmetry than perpendicular to it. This can be explained by looking at the cost functions: For the point and flat probes, the circle descriptors involve three parameters $(x, y$, and $r)$, where negative displacements of $(x, y)$ along the arc's axis of symmetry coupled with increases in radius (r) will cause the least change in cost. For the V-probe, $(x, y)$ are found to minimize the summed distances to the radial data lines (set of bisectors), so displacements in the direction 'most parallel' to the average data line (ie. along the arc's axis of symmetry) will cause the least change in cost. The SD of the flat probe's centre estimate distribution is $-15 \%$ lower than that of the point probe, but the V-probe's distributions showed further reductions of $35-50 \%$ in SD, showing that it is markedly less sensitive to measurement noise. Increased V-probe angles $(\beta)$ produced significantly lower standard deviations of the point location along the axis of symmetry but had no significant effect perpendicular to the axis of symmetry.

On the condyle image, all three probes again showed maximum SD along the axis of symmetry of the best fit arc. There is a small difference in SD between the point 
and flat probes, although this time the point probe has the smallest value. As on the true arc, the $V$-probes with $\beta$ in the range of $130^{\circ}$ to $160^{\circ}$ showed SD's up to $50 \%$ lower than the point and flat probes along both axes and showed less pronounced variation along the axis of symmetry, creating a more circular cloud of points.

The mean centre point estimated by the point probe was located about $3 \mathrm{~mm}$ from those estimated by the flat and V-probes (Figs. 4 \& 5). It appears that this difference is caused by the near flat region over the first half of the scanned range in this particular contour. The point probe will record many points on this flat region, forcing the best fit circle downwards and to a greater radius. In contrast, contact points for the flat and V-probes will move counterclockwise onto the downward slope of the contour earlier in the scanned range (assuming the probe surfaces are large enough), reducing the influence of the flat region.

\subsection{Conclusions}

The V-shaped probe design enables us to locate a characteristic feature of condylar geometry (i.e., the centre of a best fit arc) with up to half the variability of a flat probe or conventional point probe. The variability of this localization is greatest along the arc's axis of symmetry. We are planning to extend this study to three dimensions, where the V-probe will be replaced by a three-faced pyramidal probe. Such a probe is also likely to have benefits in other registration applications.

\section{Acknowledgements}

We thank Dr. Boris Flak of the UBC Department of Radiology for help in obtaining the MRI scan of the knee, Roger Tam of the UBC MAGIC Lab for assistance in converting the image for use in this project, and Robert W. McGraw of the UBC Department of Orthopaedics for discussions on TKR procedure.

\section{References}

1. Leitner, F., Picard.F., Minfelde, R., Schulz, H-J., Cinquin P., Saragaglia D.: Computer Assisted Knee Replacement Surgical Total Replacement. In: Troccaz, J., Grimson, E., Mosges, R. (eds): CVRMed-MRCAS '97. Lecture Notes in Computer Science, Vol. 1205. Springer-Verlag, Berlin Heidelberg New York (1997) 627-638.

2. Kurosawa, H., Walker, PS.,Abe, S.,Garg, A., Hunter, T. : Geometry and Motion of the Knee for Implant and Orthotic Design. Journal of Biomechanics (1985); 18 (7): 487 - 499.

3. Simon, D.A., Hebert, M.., Kanade T.: Techniques for Fast and Accurate Intra-Surgical Registration. In: DiGioia, A.M., Kanade, T.,Taylor, R. (eds.): MRCAS '94, Vol. 1. Pittsburgh, Pennsylvania. (1994) 90-97. 